AperTO - Archivio Istituzionale Open Access dell'Università di Torino

\title{
Allégorie et tautologie : la politique du poème, de Baudelaire à Mallarmé
}

\section{This is the author's manuscript}

Original Citation:

Availability:

This version is available http://hdl.handle.net/2318/1834710

since 2022-01-22T18:50:30Z

Published version:

DOI:10.3917/rom.152.0109

Terms of use:

Open Access

Anyone can freely access the full text of works made available as "Open Access". Works made available under a Creative Commons license can be used according to the terms and conditions of said license. Use of all other works requires consent of the right holder (author or publisher) if not exempted from copyright protection by the applicable law. 


\section{Allégorie et tautologie : la politique du poème, de Baudelaire à Mallarmé}

Comment penser politiquement la littérature sans réduire à un simple prétexte la singularité des œuvres, sans sacrifier, une fois encore, la spécificité du discours littéraire ? La réponse à cette question pourrait bien dépendre d'un simple constat: la littérature ne dispose jamais d'une alternative entre engagement et indifférence, dans la mesure où elle ne peut pas être apolitique. Comme tout acte de communication, l'œuvre actualise, propose et critique des normes de partage du sens, des rapports de force, une éthique ; comme toute parole, elle configure un horizon, une perspective selon lesquels penser de façon critique la communauté vivante qu'elle instaure et défait tout à la fois. C'est dire que, d'une manière ou d'une autre, à l'instar de la pensée et de l'action politiques, la littérature, en tant qu'elle engage toujours une pragmatique et une rhétorique, est une économie de la violence. On comprendra alors que Jean Bessière ait pu décrire en termes juridico-politiques le fonctionnement de la communication littéraire. Sa caractérisation du paradigme romantique comme littérature à statut d'exception, notamment, invite à une interprétation de la littérature dans les termes d'une pensée du pouvoir nourrie des théories de Giorgio Agamben ${ }^{1}$ Penser la littérature oblige donc à dégager les modalités politiques qui la constituent non pas circonstanciellement mais de façon intrinsèque. Dans cette perspective, les discussions théoriques autour du romantisme et le débat des poètes avec le paradigme romantique de la création littéraire ne peuvent être réduits au XIX ${ }^{\mathrm{e}}$ siècle à une futile querelle d'esthètes. C'est en tout cas ce que nous tenterons de montrer ici à travers l'étude des rapports complexes qui lient deux ouvres cruciales pour le renversement critique de

1. Voir pour l'exposé le plus complet dans son œuvre de la notion d'exception, Giorgio Agamben, Stato di eccezione, Bollati Boringhieri, 2003. 
ce qui fut au cœur de la doctrine romantique de l'œuvre d'art, à savoir la théorie du symbole.

Significativement, Baudelaire et Mallarmé, quoique de manières fort différentes, se démarquent du modèle romantique par une utilisation concertée de l'allégorie. Significativement aussi, le dialogue posthume avec Baudelaire que constituent les Poésies de 1887 et certains textes des Divagations semble se focaliser à plusieurs reprises sur la dimension politique de la forme allégorique, sur sa capacité à fonder la possibilité d'un espace commun d'existence. Après un bref rappel de la théorie romantique du symbole, nous tenterons d'expliciter le sens de l'utilisation baudelairienne de l'allégorie relativement à cette théorie. Nous aurons alors en main les prémisses nécessaires à la compréhension de la critique mallarméenne de l'allégorie baudelairienne : il faudra alors en étudier le détail à travers une lecture comparée de quatre textes où l'utilisation de l'allégorie se voit directement articulée à une interrogation sur les fondements symboliques de la communauté humaine. Peut-être pourrons-nous alors indiquer quelques éléments d'une « critique de la violence » en littérature.

\section{DÉFINITION RHÉTORIQUE DU SYMBOLE ROMANTIQUE}

Par symbole il faut entendre une certaine économie du rapport entre représentation et présentation. On retrouve ici l'origine kantienne de la question romantique comprise comme question du sujet. Comme le notaient Philippe Lacoue-Labarthe et Jean-Luc Nancy, la pensée romantique s'enracine dans la critique du sujet substantiel accomplie par Kant. Parce qu'il est une forme vide, le sujet kantien est un «sujet imprésentable 2 .» Or cette imprésentabilité qui dépossède le sujet de lui-même, laisse place, chez les romantiques, à l'" autorité ${ }^{3}$ "d'une œuvre-sujet ${ }^{4}$ qui se présente soi-même et à soi-même. L'autorité de l'œuvre semble donc se construire sur un oubli du fait qu'en elle subsiste une part imprésentable. Cela constitue sans doute la croyance propre au mouvement romantique, son point aveugle. On peut alors comprendre la définition rhétorique que Jean Bessière donne du symbole : «signe complexe qui joue d'une présentation et d'une représentation, qui laisse dans l'équivoque le rapport de la présentation à la représentation, et qui peut, en conséquence, se lire selon l'évidence de la présentation,

\footnotetext{
2. Philippe Lacoue-Labarthe et Jean-Luc Nancy, L'Absolu littéraire, Le Seuil, 1978, p. 43.

3. Ibid., p. 48

4. Ibid., p. 47.
}

2011-Non spécifié

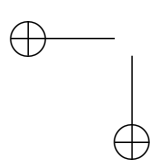


selon celle de la représentation, sans que l'articulation de l'une et de l'autre soit résolutoire ${ }^{5}$. C'est là une définition fonctionnelle de ce que Philippe Lacoue-Labarthe et Jean-Luc Nancy nomment " l'équivoque romantique ${ }^{6}$ " en même temps que la possibilité d'une critique politique des définitions romantiques de l'œuvre et de la littérature. En méconnaissant le caractère non évident de ce qui fonderait l'œuvre en vérité, qu'on le nomme le sujet, l'art, le poème ou la critique, le premier romantisme manquerait la nécessaire problématisation du fait littéraire sans laquelle la transmission dialogique d'un sens commun ne peut se poursuivre. "L'équivoque romantique " est ainsi paradoxalement une pensée de l'évidence et de l'autonomie de l'œuvre qui produit un écrasement de ses pôles rhétoriques (ethos, pathos, $\log o s)^{7}$ en une totalité inconditionnée.

\section{ALLÉGORIE ET THÉOLOGIE DE LA SÉPARATION : BAUDELAIRE ET BENJAMIN}

On peut dès lors caractériser l'allégorie baudelairienne relativement à la doctrine romantique. Tout en s'appuyant explicitement sur la théorie benjaminienne qui définit "moins un trope qu'une vision du monde propre au mélancolique ${ }^{8}$ ", Patrick Labarthe a pu établir une typologie précise 9 du phénomène allégorique chez Baudelaire, se donnant ainsi les moyens d'en approcher le détail, à travers la multiplicité des éléments textuels qui y participent. Le critique a montré en quoi la poétique baudelairienne de l'allégorie puisait à trois grandes sources (Chateaubriand, la tradition des prédicateurs, les théoriciens de la Providence divine) desquelles Baudelaire retient surtout l'affirmation d'une distance entre sens et réalité, tout en refusant opiniâtrement ce qui la viendrait combler de façon trop optimiste. L'allégorie telle qu'il la pratique relève alors d'une théologie de la séparation.

P. Labarthe confirme ainsi l'intuition de Benjamin. Dès sa thèse sur le drame baroque allemand (Ursprung des deutschen Trauerspiels), ce dernier entreprend une réhabilitation de l'allégorie baroque contre la dimension

\footnotetext{
5. Jean Bessière, La Littérature et sa rhétorique, PUF, 1999, p. 6.

6. L'Absolu littéraire, ouvr. cité, p. 419-433.

7. Voir J. Bessière, Quel statut pour la littérature, PUF, 2001, p. 17-24.

8. Patrick Labarthe, Baudelaire et la tradition de l'allégorie, Droz, 1999, p. 60.

9. Cette typologie distingue l'emblème (juxtaposition d'un signifiant iconique et d'un signifiant verbal lui-même dissocié en une légende - motto - et une épigramme - subscriptio), les allégories narratives et figuratives et les allégories fondées sur une oscillation énonciative entre intériorisation et détachement (ibid., p. 46-55).
} 
immanentiste du romantisme. L'allégorie témoignerait d'une séparation, sa temporalité serait celle de l'histoire et du dépérissement, quand le symbole n'advient que dans le présent ébloui de l'instant mystique ${ }^{10}:$ " Alors que dans le symbole, par la sublimation de la chute, le visage transfiguré de la nature se révèle fugitivement dans la lumière du salut, en revanche, dans l'allégorie, c'est la facies hippocratica de l'histoire qui s'offre au regard du spectateur comme un paysage primitif pétrifié. " Dans son achèvement ${ }^{11}$, une telle pensée annonce une rupture radicale avec l'expressivisme et l'anthropologie de la souveraineté humaine sur quoi se fonde la pensée romantique de l'œuvre littéraire. De fait, cette conception de l'allégorie récuse la sacralisation romantique de la totalité symbolique, contraignant le lecteur "à revenir des synthèses idéalistes factices ${ }^{12}$ ", au profit d'une théologie de la séparation lourde d'implications quant aux modalités de partage du sens qu'elle inaugure.

\section{INTERPRÉTATION RHÉTORIQUE DE L'ALLÉGORIE BAUDELAIRIENNE}

Fonder sa poétique sur une théologie de la séparation, c'est donc pour Baudelaire reconnaître l'absoluité d'un autre que l'écriture ne maîtrise pas ; c'est aussi, et par là même, impliquer une problématicité de l'œuvre, de la présentation qu'elle constitue et des éléments formels et informationnels qu'elle rassemble, mesurés à l'aune de cet autre à la fois commun et inaccessible. C'est en même temps indiquer clairement la présomption d'une pertinence de l'œuvre (à travers la référence à un absolu de sens, même si celui-ci reste problématique et retiré). Notons encore que la présentation allégorique installe ses données informationnelles dans une insuffisance partielle : elle les littéralise au point de les rendre absolument vaines et insignifiantes (c'est l'indication de l'ennui ou de l'amer savoir qui vient au terme du "Voyage ») ; elle les délittéralise en faisant de cette littéralité déconcertante l'occasion d'un questionnement d'une ampleur hypothétiquement nouvelle (messianique). Se lit ici l'inversion des institutions sociales et linguistiques de la société en même temps que l'annonce d'un lieu commun

10. Walter Benjamin, Origine du drame baroque allemand, trad. S. Muller, 1974, 1985, Flammarion, p. 178.

11. Achèvement que manque peut-être Benjamin dans la mesure où il continue de caractériser l'allégorie comme expression. Voir à propos de cette critique de Benjamin, Catherine Perret, «L'allégorie : une politique de la transmission ? », Europe n 804, avril 1996, p. 102-112.

12. Guy Petitdemange, «Savoir, connaître. Notes autour du Trauerspiel », dans Walter Benjamin - Critique philosophique de l'art, Rainer Rochlitz et Pierre Rusch (dir.), PUF, 2005, p. 161.

2011-Non spécifié

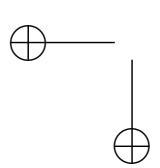




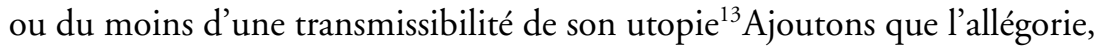
en tant que jeu sur le maintien et la ruine de la convention ${ }^{14}$, se donne explicitement comme transmission, impliquant une socialité du texte et des savoirs. À ce titre, elle échappe au mythe du commencement absolu qui préside peu ou prou à la pensée du symbole ${ }^{15}$ De plus, la convention, du fait qu'elle peut apparaître comme désignation transparente de l'Idée, risque toujours par là même de s'opacifier. Il y a ici un jeu sur la clarté et sur l'énigmaticité du signifiant allégorique, énigmaticité qui est à penser alors comme déshérence du signifiant : ainsi de la "Fontaine de sang ", où les tercets semblent interpréter allégoriquement l'image proposée dans les quatrains, sans pourtant qu'un rapport d'évidence s'établisse pour le lecteur entre l'image et sa possible interprétation ${ }^{16}$

L'allégorie baudelairienne excède donc par deux fois la tautologie autosuffisante de l'œuvre symbolique: par son appartenance explicite (mais parfois problématique) à une convention qui en est, formellement, la condition de possibilité ; par la désignation de la séparation de sa pertinence et de la forme signifiante conventionnelle. Au plan politique, un tel complexe communicationnel implique une logique fondée sur le partage d'un ensemble de conventions héritées, d'une interprétation du monde par la transcendance et d'une attente qu'on pourrait qualifier d'utopiste.

\section{LA CRITIQUE MALLARMÉENNE DE BAUDELAIRE : DU CYGNE AU SIGNE, DU « VIEUX SALTIMBANQUE » À « LA DÉCLARATION FORAINE »}

On le devine, l'athéisme de Mallarmé s'accommode mal de la perspective théologique de l'allégorie baudelairienne. De fait, il semblerait que les Poésies soient un adieu progressif à Baudelaire, dont l'ombre rayonne sur un grand

13. «Avis aux non-communistes : Tout est commun, même Dieu », peut-on lire dans Mon Cœur mis à nu (Fusées, Mon cœur mis à nus, La Belgique déshabillée, édition d'André Guyaux, Gallimard, 1986, p. 112).

14. Qu'elle soit attestée ou mimée, la convention préside au déploiement de l'allégorèse que déclenchent des marqueurs allégoriques identifiables - majuscule, thématique de la mort et de la vanité.

15. Voir cette formule de Novalis citée par W. Benjamin : «Existe-t-il un art d'invention sans données, un art d'invention absolu ? »(Le Concept de critique esthétique dans le romantisme allemand, Champs essais, 1986, (première impression du texte allemand en 1919), p. 106).

16. Voir Baudelaire et la tradition de l'allégorie, ouvr. cité, p. 53. 
nombre de textes, avant de venir " se rasseoir/Contre le marbre vainement ${ }^{17}$ " de sa stèle. Nous mesurerons l'importance du remaniement mallarméen de l'allégorie en explicitant la dimension polémique de deux poèmes allégoriques de Mallarmé, "Le vierge, le vivace et le bel aujourd'hui ${ }^{18}$ " (1885) et "La Déclaration foraine ${ }^{19}$ " (1887), où s'articulent les fondements de sa condamnation de la poétique baudelairienne.

Dans un commentaire extrêmement rigoureux ${ }^{20}$, Jean-Claude Milner donne du poème " Le vierge, le vivace et le bel aujourd'hui ${ }^{21}$ " l'interprétation suivante : le premier quatrain, exclamatif, dit au discours direct la posture hugolienne d'une croyance au lendemain dont tout pourra advenir, «de telle sorte que tout jour puisse être celui-là ${ }^{22}$ "; le premier tercet exprime dans un récit rétrospectif le moment de Baudelaire, qui consiste en l'attente d'un tel jour accompagnée du savoir qu'il n'adviendra jamais. Ce moment est une manière de tragédie allégorique dans laquelle le monde ne désigne que l'absence du divin ${ }^{23}$. Là où les deux poètes se rejoignent selon Stéphane Mallarmé, c'est dans leur positionnement par rapport à ce jour vivace et beau, que les idéologies révolutionnaires n'avaient cessé de promouvoir : l'un sous la forme de la confiance, l'autre par la prostration endeuillée ${ }^{24}$ Tous les deux sont victimes d'une même illusion : que le sens se constitue relativement à un idéal d'incarnation de l'idée, dont le nom est l'Idéal ${ }^{25}$. Ainsi, selon J.-C. Milner, « le Cygne » fantomatique du dernier vers est-il Stéphane Mallarmé lui-même, dont l'attitude est celle d'un retranchement jugé méprisable, car improductif, par le contemporain. Sans doute le critique a-t-il raison de voir en ce texte un "Tombeau de Stéphane Mallarmééc ", lequel prend sur lui, par la décision finale du dernier tercet, la mort définitive de l'Idéal. Pourtant, nous objecterons à la généralisation de cette décision

17. «Le Tombeau de Charles Baudelaire », Poésies, Euvres complètes, éd. de Bertrand Marchal, Gallimard, collection La Pléiade, 1998, t. I, p. 39.

18. Poésies, ouvr. cité, ibid., p. 36.

19. Poèmes en prose, éd. citée, p. 423.

20. Jean-Claude Milner, Mallarmé au tombeau, Verdier, 1999.

21. Poésies, éd. citée, p. 36.

22. Mallarmé au tombeau, ouvr. cité, p. 56.

23. Ibid., p. 57.

24. Baudelaire, en 1852, avait abandonné toute idée démocratique.

25. Mallarmé au tombeau, ouvr. cité, p. 70.

26. Ibid., p. 56.

2011-Non spécifié 
pour l'ensemble de la poétique mallarméenne $e^{27}$. L'auteur des «Éventails " et du " Billet " à Whistler, n'est certainement pas nihiliste.

En effet, le dialogue avec Baudelaire n'est pas clos au terme du sonnet de 1885. Si l'auteur y dialoguait avec «Le Cygne» (1860), texte dédié à Hugo (où, pour le narrateur baudelairien, tout, dans Paris, devenait allégorie $\left.{ }^{28}\right)$, et, récapitulant l'opposition de l'un à l'autre, se distinguait des deux par une immobilité fantomatique, il est excessif d'y voir simplement l'expression de l'idée que rien n'a lieu. Il n'y a pas qu'une position négative dans ce sonnet. Signalé par une majuscule inattendue, un élément positif y surgit : « le Cygne ». La distance qui sépare Baudelaire de Mallarmé n’est pas, comme le pense J.-C. Milner, celle qui sépare uniquement le deuil de l'Idéal de l'abdication de ce deuil, mais celle qui distingue une poétique orientée théologiquement d'une pratique radicalement sceptique de la poésie.

La discussion avec Baudelaire n'en reste pas au niveau des principes, sans quoi elle resterait, par une capture dialectique, prise au piège de l'Idéal, dans les lacs d'une façon de poser la question du discours poétique selon la problématique de ces principes mêmes : la décision de Mallarmé est sa poésie, différente de celle de Baudelaire. C'est en tout cas ce que nous voudrions démontrer.

Rappelons dans un premier temps que «Le vierge, le vivace et le bel aujourd'hui » s'oppose point par point au "Cygne» des Fleurs du Mal ${ }^{29}$. Or le Cygne de Baudelaire s'est échappé d'une baraque de foire. « Le vieux Paris n'est plus ", affirme le locuteur, et il ajoute :

Je ne vois qu'en esprit tout ce camp de baraques,

Ces tas de chapiteaux ébauchés et de fûts,

Les herbes, les gros blocs verdis par l'eau des flaques,

Et, brillant aux carreaux, le bric-à-brac confus.

Là s'étalait jadis une ménagerie ;

Là je vis, un matin, à l'heure où sous les cieux

Froids et clairs le Travail s'éveille, où la voirie

Pousse un sombre ouragan dans l'air silencieux,

Un cygne qui s'était évadé de sa cage,

27. Ibid., p. 77 : «Un moment arrive toujours où l'on est tenté de dire : rien n'a eu lieu. C'est le moment mallarméen. » Remarquons que Mallarmé ne dit jamais : rien n'a eu lieu ; mais : « rien n'aura eu lieu que le lieu ». Ce qui est différent.

28. «Le Cygne », Les Fleurs du Mal, Euvres complètes I, éd. de Claude Pichois, Gallimard, coll. «Bibliothèque de la Pléiade », 1975, p. 85-87 : «Paris change ! mais rien dans ma mélancolie/N'a bougé ! palais neufs, échafaudages, blocs,/Vieux faubourgs, tout pour moi devient allégorie,/Et mes chers souvenirs sont plus lourd que des rocs ».

29. Voir Jean-Claude Milner, ouvr. cité, p. 43-47. 
Et, de ses pieds palmés frottant le pavé sec,

Sur le sol raboteux traînait son blanc plumage.

Près d'un ruisseau sans eau la bête ouvrant le bec

Baignait nerveusement ses ailes dans la poudre ${ }^{30}$.

La baraque désertée ne laisse pas d'évoquer celle du vieux saltimbanque du Spleen de Paris. De fil en aiguille, c'est « La Déclaration foraine " datant de 1887 qui est convoquée. D’où la proposition suivante : dans ce texte postérieur à " Le vierge, le vivace et le bel aujourd'hui », Mallarmé reprend sa réflexion sur les rapports de Baudelaire à Hugo, en opposant plus nettement le caractère positif de ce qui le distingue de ses précurseurs, chose que la fin ambiguë du sonnet avait pu masquer. Dans "Le Vieux Saltimbanque ${ }^{31}$ ", texte daté de 1862, le narrateur se promène dans une foire qu'il décrit comme un "jubilé populaire " où l'odeur de friture a le rôle de "l'encens". Mais il ne faut pas s'y tromper : cette fête vécue quasi religieusement par le peuple n'amène pas l'exaucement de ses souhaits les plus chers; tout au plus est-elle un répit, l'oubli temporaire des maux quotidiens ${ }^{32}$ De fait, Baudelaire prend soin de nous décrire la religion démocratique (ou ce qui en tient lieu ici) comme une gigantesque confusion où les cris se mêlent aux odeurs de friture. Rien de moins rousseauiste ${ }^{33}$, en somme, que ce chaos social, cette orgie économique qui sert de toile de fond à la plus sinistre manifestation de la misère humaine. C'est dans cette atmosphère de confusion que la découverte de la détresse du vieux saltimbanque peut précipiter le sens de la scène dans une dimension clairement catastrophique, conduisant à l'allégorèse finale du narrateur baudelairien :

Et, m'en retournant, obsédé par cette vision, je cherchai à analyser ma soudaine douleur, et je me dis : Je viens de voir l'image du vieil homme de lettres qui a survécu à la génération dont il fut le brillant amuseur ; du vieux poète sans amis, sans famille, sans enfants, dégradé par sa misère et par l'ingratitude publique, et dans la baraque de qui le monde oublieux ne veut plus entrer ${ }^{34}$ !

30. Les Fleurs du Mal, éd. citée, p. 85.

31. Le Spleen de Paris, Euvres complètes I, éd. Claude Pichois, Gallimard, coll. «Bibliothèque de la Pléiade », 1977, p. 295.

32. «En ces jours-là il semble que le peuple oublie tout, la douleur et le travail ; il devient pareil aux enfants. Pour les petits c'est un jour de congé, c'est l'horreur de l'école renvoyée à vingt-quatre heures. Pour les grands c'est un armistice conclu avec les forces malfaisantes de la vie, un répit dans la contention et la lutte universelle » (ibid.).

33. Sur l'idéal harmonique de la pensée politique de Rousseau, on pourra se reporter à l'article de Jean Starobinski, «Le remède dans le mal : sur la pensée de Rousseau », Le Remède dans le mal, Gallimard, 1989, en particulier à la section II intitulée « Socialité de la musique », p. 208-232.

34. Ibid.

2011-Non spécifié 
Le poème propose donc une interprétation complexe de la place du poète dans la société : d'une part, le poète vieillissant est mis au ban de la société et de la célébration festive de soi qu'elle s'offre complaisamment; d'autre part, le poète moderne, qui se trouve non moins exclu de cette célébration, est tenu d'en dévoiler la dimension à la fois factice et catastrophique. Pour le dire autrement, de la passivité désespérée du vieux saltimbanque à la non moins passive rêverie réflexive du narrateur, l'unique différence tient à la production d'une pensée allégorique et catastrophique du réel. Sartre y insistait: l'action n'est pas une catégorie baudelairienne ${ }^{35}$. Le texte se termine sur la velléité d'une aumône, qu'un mouvement de foule empêchera. L'allégorisme baudelairien, en même temps qu'il se donne pour un fantastique instrument de subversion idéologique, ne semble jamais très éloigné d'une démission résignée devant l'inertie du monde réel.

Au-delà des nombreuses similitudes indiquant un dialogue concerté entre les deux textes, le contraste entre " Le Vieux Saltimbanque » et « La Déclaration foraine ${ }^{36}$ " est net. Le poète avec "Madame... seule tu sais Qui " arrive par hasard au milieu d'une fête, qui, comme toutes les fêtes des Divagations, relève du mystère ${ }^{37} \mathrm{Il}$ s'agit donc encore d'un poème de la religion démocratique. Comme chez Baudelaire la fête est un temps d'" exception à la misère ". Comme Baudelaire, Mallarmé insiste sur le tumulte qu'elle engendre. Comme lui, il rencontre un pauvre vieillard dont la baraque délabrée semble ne receler aucune attraction. Comme lui, il éprouve de la compassion pour sa misère. Cependant son attitude vis-à-vis du vieillard est totalement différente. Au lieu d'une rêverie mélancolique sur la ruine des carrières littéraires, cette rencontre débouche sur une action efficace en faveur du saltimbanque déchu. En effet, dans la baraque " apparemment vide $^{38}$ ", la compagne du poète décide d'improviser un spectacle qui sera sa simple exposition redoublée (tautologiquement, semble-t-il) d'un poème.

Le narrateur mallarméen et son amie s'opposent donc clairement au solitaire dandy baudelairien, réduit à une passivité devant l'appréhension d'un vide qui le terrifie sans doute : Qu'il n'y ait rien dans la baraque, soit ; mais qu'on y donne un spectacle! De même que le vieux saltimbanque désignait peut-être Lamartine, comment ne pas reconnaître dans le vieillard

35. Jean-Paul Sartre, Baudelaire, Gallimard, 1947, p. 52.

36. Divagations, ouvr. cité, p. 93-98.

37. Ibid., p. 425 : le pré, « quand l'institue le mot mystérieux de fête » prend un « caractère frérial d'exception à la misère ».

38. Ibid., p. 424. 
de "La Déclaration foraine ", qui reste incapable de montrer «l'hallucination d'une merveille ${ }^{39}$ ", et ne présente que "l'inanité de son famélique cauchemar ${ }^{40}$ ", le chroniqueur en somme stérile du "Vieux Saltimbanque ", voire Baudelaire lui-même. En outre, le texte de Mallarmé s'oppose jusque dans sa forme à celui de Baudelaire : tel un écrin, il contient le joyau d'un poème ("La chevelure vol d'une flamme ») que Baudelaire, médusé par la profondeur de la misère, n'a pas enfanté. Confronté à l'absence d'un sens politique instituant, contre une mélancolie qui s'exprime de façon privilégiée dans la forme subversive mais inefficace de l'allégorie, Mallarmé invente une "bravoure ${ }^{41}$ " poétique dans la valorisation active de ce qui, dans la célébration maladroite des fêtes populaires, contient la trace d'une vocation de l'homme à la divinité.

Or la posture mallarméenne s'affirme ici dans toute son originalité comme une nouvelle poétique de l'allégorie. "La Déclaration foraine " est sans conteste un poème allégorique. Selon deux niveaux au moins. Au niveau global d'abord, la scène est à entendre comme une allégorie de la position du poète quant à la question des fondements religieux de la société, du sens auquel vouer la communauté humaine ${ }^{42}$. À un niveau plus local ensuite, Mallarmé prend soin de l'indiquer au lecteur ${ }^{43}$, le spectacle donné par le poète et sa compagne est de nature allégorique. Cependant il semble que cette dimension allégorique renouvelle de façon radicale le paradigme baudelairien. Au niveau local, la "vivante allégorie " mallarméenne n'affirme d'autre transcendance que celle de sa propre présentation. La figure féminine en effet, " ne mouvant astre ni feu au doigt ", c'est-à-dire sans l'appui d'attributs propres à désigner une idée qui la dépasserait, n'est qu'elle-même. De même, le dispositif de redoublement de l'apparition féminine par le sonnet consiste en une affirmation de son identité. Cela se formule comme un paradoxe : d'une part l'allégorie est une tautologie; d'autre part réflexivement le poème est à la fois une allégorie de la tautologie, et l'affirmation d'une tautologie de l'allégorie. Pour l'interprétation globale du texte, cela interdit toute démission du poète devant la réalité. L'allégorie est un fait. Comme tel, elle est une part de la réalité à partir de laquelle elle s'élabore. L'interprétation
39. Ibid.
40. Ibid.
41. Ibid. p. 94.

42. À ce titre il faut noter la dimension allégorique des protagonistes de l'histoire : « Madame seule tu sais qui », Idée du poète, le vieillard, etc. On verra à ce sujet l'étude de Bertrand Marchal dans La Religion de Mallarmé, Corti, 1988, p. 258-271.

43. « Mon aide à la taille de la vivante allégorie [...] » (« La Déclaration foraine »). 
de la scène de foire en termes allégoriques n'amène pas à la constatation du manque de ce à quoi la foule aspire. L'instinct de ciel en chacun n'est pas la promesse messianique d'un futur meilleur mais la preuve factuelle de sa possibilité, même si, dans la mesure où il peut rester ignoré, il n'est pas une condition suffisante à l'établissement d'un ordre nouveau : c'est ce qui justifie l'action du poète.

Cette reconfiguration de l'allégorie est centrale dans la poétique mallarméenne. Outre le fameux "sonnet allégorique de lui-même ", elle permet d'entendre des textes tels que "À la nue accablante tu » ou " Le Démon de l'analogie ${ }^{44}$ ".

Congédiant la problématique d'une séparation du réel et de l'Idéal, le poète ne sombre ni dans le nihilisme, ni dans une restauration du paradigme romantique selon lequel l'idéalité du monde (c'est-à-dire de la conscience) lui serait immanente, resterait en son pouvoir. Ce qui le distingue de Baudelaire, ce n'est pas tant la mort du cygne, l'abdication définitive d'une figure du poète vouée à l'idéal, que l'avènement du Cygne comme Idée reconnue dans sa factualité contingente et non plus sous les espèces d'une totalité souveraine comme c'était le cas dans le premier romantisme. Le sonnet qu'analyse J.-C. Milner est donc le préalable d'une poésie qui s'accommode du rien parce qu'elle a reconnu la positivité d'un fait. Que cette reconnaissance relève en outre d'un scepticisme et non de la substitution d'une doctrine à une autre, cela semble assez clair, dans la mesure où dépasser la question de l'Idéal, c'est dépasser aussi l'horizon de la vérité, d'un fondement ontologique du sens.

\section{INTERPRÉTATION RHÉTORIQUE DE L'ALLÉGORIE MALLARMÉENNE}

La refonte mallarméenne de l'allégorie entraîne une redéfinition profonde de son fonctionnement rhétorique et donc de ses implications politiques. Parce qu'il maintient la dimension allégorique de son texte, Mallarmé

44. Nous nous contenterons d'indiquer quelques pistes de lecture pour ce dernier texte. « Des paroles inconnues chantèrent-elles sur vos lèvres, lambeaux maudits d'une phrase absurde ? » Cette phrase («La Pénultième est morte ») qu'évoque « Le Démon de l'analogie » (Divagations, ouvr. cité, p. 86-88), n'offre pas son élucidation selon les règles discursives usuelles. Pour comprendre l'énoncé « La pénultième/Est morte » il faut entendre le mot «pénultième » à la fois en mention et en usage. Ce phénomène redouble de façon inverse l'architecture narrative du poème. En effet, la phrase mystérieuse d'abord présentée pour elle-même, mentionnée, trouve un répondant dans le réel en cet instrument $v u$ dans la vitrine d'un luthier (auquel, peut-être, manque l'avant-dernière corde). D'abord mention, elle fait alors sens à être prise en usage. 
continue d'inviter le lecteur à la reconnaissance d'une altérité par rapport à quoi celui-ci prend sens. Pourtant, cette altérité n'est plus que celle du texte lui-même : le sens allégorique du poème, dans la mesure où il se réduit à une tautologie, ne peut être pensé comme séparé. Du coup, si la "vivante allégorie » que restitue le texte a pour seul contenu l'affirmation d'une présence, il n'y a plus lieu d'en faire le moyen d'une justification métaphysique et partant, autoritaire du monde, à laquelle nous invitait encore Baudelaire sur le mode du dénigrement ${ }^{45} \mathrm{C}^{\prime}$ est placer le poème sous un nouveau régime de problématicité et de circulation du sens. Le sens pour Mallarmé n'est plus nostalgie d'une transcendance irrémédiablement éloignée, mais l'objet d'un constat, ce qui n'empêche pas, au moins formellement, le maintien d'une convention, d'une communicabilité. Cela le distingue d'ailleurs aussi bien de l'auteur des Fleurs du Mal que des romantiques. En effet, la tautégorie romantique, ce pouvoir qu'aurait eu l'œuvre de relever de sa propre catégorie, se résume chez Mallarmé à une tautologie. L'œuvre est ce qu'elle est, mais elle n'est aucunement dans une relation de pouvoir exclusif vis-à-vis de son sens, dans la mesure où, purement factuel (et non pas essentiel, définitoire d'une essence), le sens y est soumis à une contingence irréductible. Le dispositif réflexif chez Mallarmé invite donc à une reconnaissance de la contingence de l'œuvre et du sens, là où la réflexivité romantique était affirmation d'un pouvoir indiscutable.

D'un point de vue politique, l'affirmation de la contingence du poème entraîne une mise en cause des institutions sociales et linguistiques de la réalité non plus en les subvertissant ironiquement comme chez Baudelaire mais parce qu'elle en révèle la pièce principale ou rien, l'absence de justification. Quand l'altérité de l'Idéal impliquait une problématisation de la communication à partir d'une utopie irrémédiablement reculée, le questionnement mallarméen de la réalité n'a plus rien d'utopique et s'effectue au cœur du réel et de la communication, dans la mesure même où c'est l'absence de justification du réel et des normes de partage du sens (dont la possibilité reste cependant, in extremis, maintenue) qui le suscite et en constitue l'objet.

45. Encore une fois, les remarques de Sartre sur Baudelaire se révèlent fondamentales : l'idée du péché est une manière d'entériner dogmatiquement l'existence du monde tel qu'il est, selon des cadres métaphysiques indiscutés qui fournissent au pécheur une place, une justification. « Le coupable a sa fonction dans un univers théocratique. Sa fonction et ses droits : il a droit au blâme, au châtiment, au repentir. Il concourt à l'ordre universel et sa faute lui confère une dignité religieuse, une place à part dans la hiérarchie des êtres : il est à l'abri sous un regard indulgent ou courroucé » (ouvr. cité, p. 68).

2011-Non spécifié 


\section{POLITIQUES DE L'ALLÉGORIE : VERS UNE « CRITIQUE DE LA VIOLENCE » EN LITTÉRATURE}

Comme toute œuvre de langage dont la dimension critique est explicite, les poèmes de Baudelaire et de Mallarmé élaborent une économie de ce qui se tient en retrait de la norme, qui en précède l'imposition, ou l'empêche simplement de s'établir, une économie de la violence. Les concepts utilisés par Giorgio Agamben dans Stato di eccezione seront ici d'une grande utilité. Il nous faut en faire un bref rappel. Dans cet essai, le philosophe étudie les rapports entre le droit et son application. Après avoir montré comment l'application du droit passe nécessairement par sa suspension, l'auteur remarque que le juridique semble ne pouvoir subsister qu'à travers une capture de l'anomie qui permet cette application. Il y aurait alors, du fait de cette intégration de la violence dans l'ordre juridique, le risque d'une normalisation de l'état d'exception où l'acte le plus arbitraire a force de loi. Or, cette configuration du juridique est consubstantiellement une configuration ontologique ${ }^{46}$

Cette configuration engage donc avec elle, et c'est ce qui nous intéresse ici, tout acte de communication. On comprend alors que l'entente de cette zone d'anomie, la compréhension de ce moment de suspension soient fondamentaux. Giorgio Agamben convoque pour cela la pensée benjaminienne de la violence rappelant que le penseur allemand oppose deux types de violence : une violence mythico-juridique et une violence pure $^{47}$ La violence mythico-juridique se tient toujours dans une relation au droit, elle se donne comme moyen de l'application du droit, son résultat est l'installation d'un pouvoir. La violence pure, en tant qu'elle ne se définit plus comme moyen de l'application du droit, mais comme relation à sa propre médialité [sic], est un autre nom de l'action humaine une fois rompu le lien entre violence et droit $^{48}$

La définition romantique de l'œuvre d'art installait le poème dans une zone d'anomie qui est, à la fois et selon la même autorité, suspension et imposition de sa propre norme. L'art tend alors à se fonder sur le

46. G. Agamben, Stato di eccezione, ouvr. cité., p. 78 : « La relation entre norme et réalité implique la suspension de la norme, de la même manière que, dans l'ontologie, la relation entre langage et monde implique, sous la forme d'une langue [en français dans le texte] la suspension de la dénotation » (notre traduction).

47. Ouvr. cité, p. 78-80.

48. Voir W. Benjamin, « Critique de la violence », Euvres I, Gallimard, 2000, p. 210-243. Le texte original est de 1921. 
moment d'une tension métaphysique fondamentale où la norme se voit suspendue pour pouvoir être imposée, où le sens, pour apparaitre, est soustrait à lui-même, tandis que la présentation est donnée selon l'évidence de son intégration au sens. Ce moment est donc identique à celui où l'action violente, revendiquant son intégration juridique, se confond avec le droit pour en assurer l'instauration. Une telle communication n'est pas sans consonance, en effet, avec l'affirmation d'un état d'exception qui apparait alors comme le paradigme politique du symbole. Par violence nous entendons donc ici non pas une neutralité (quant à la norme) de l'acte qui précède la loi ou le sens, mais une relativité inextricable de la loi et de sa suspension.

Dans l'allégorie baudelairienne, la séparation du sens et de l'acte de communication semble interdire toute indécidabilité entre la norme et son actualisation. L'anomie est ici répartie sur deux plans : d'abord, l'acte de communication dans la mesure où il mobilise des signifiants insuffisants au regard du sens sous-entendu est anomique, violent ; la relation d'altérité qui relie l'anomie au sens est devenue nettement exclusive; la violence est le signe d'un échec : le sens lui reste inaccessible; ensuite, l'affirmation de cet échec, de cette exclusivité, procède elle-même d'une zone d'anomie. Mais ne peut-on pas entendre dialectiquement cette affirmation d'exclusivité ? En effet, si l'allégorie baudelairienne s'oppose frontalement au symbole, n'est-elle pas par là même comme un écho à la loi d'exception qui définit la dimension politique de ce dernier ? Dès lors il faut se demander jusqu'à quel point se recouvrent ironie et problématicité, subversion et construction du sens par une reconnaissance commune de sa problématicité. Dans quelle mesure la subversion baudelairienne se distingue-t-elle, dans la forme de communication qu'elle propose, de l'autoritarisme d'une théocratie qu'elle revendique par ailleurs ? L'exclusivité de l'acte et de la norme indique en tout cas que la question du rapport au sens continue de se poser selon les termes d'une connexion entre droit et violence, entre langue et intuition. C'est là la limite de l'allégorie baudelairienne : son héritage onto-théologique.

Chez Mallarmé, la violence ne se définit plus par rapport à une fin, à l'imposition d'un sens dans la mesure où le sens n'est plus fonction de sa réalisation : la présentation contingente de l'œuvre n'est plus affirmation d'un sens. Celui-ci est ainsi ramené à sa puissance. L'allégorie mallarméenne propose à cet égard une violence d'un type nouveau. En effet, il y a bien violence du fait que rien ne norme le discours tautologique (nous l'avons vu, l'allégorie mallarméenne est une tautologie). Mais dans la mesure où celui-ci n'est application d'aucune norme, tout en se donnant pourtant 
comme moyen pur d'une norme (c'est ce qu'implique le maintien, à vide, de la dimension allégorique du texte, un recours à la convention), il semble, en se faisant justement réflexion de sa médialité ${ }^{49}$, rejoindre cette violence pure dont parle Benjamin, violence qui ne mène à l'instauration d'aucun pouvoir. Il laisse résonner le sens gratuitement - loin pourtant de toute indifférence au monde - et sans en forcer l'imposition dans les termes viciés d'un accomplissement qui se voudrait définitif ${ }^{\varsigma_{0}}$

(Université de Toulouse II)

49. Chez Mallarmé, il ne reste de l'allégorie privée de la norme dont elle est censée se faire le moyen, que la mise en évidence pour lui-même de ce caractère de moyen, de cette médialité.

50. Cela permet de porter un nouvel éclairage sur ce que Jacques Rancière nomme l'archipoétique mallarméenne. Pour le philosophe, Mallarmé élabore dans sa réflexion politique une archipoétique équivalente à l'archipolitique (à la répartition des rôles dans la société selon un «partage du sensible ») platonicienne tout en s'en distinguant par deux aspects fondamentaux : le fondateur en est un homme du commun ; l'idée est un simulacre pur, qui ne représente plus rien que le multiple lui-même. Or, si l'idée est simulacre, si l'élu est devenu n'importe qui, il faut préserver l'idée de sa transformation tendancielle en mythe, maintenir les barrières : cela expliquerait circonstanciellement pour J. Rancière la retenue mallarméenne, son retrait, dans l'écriture comme sur la scène politique, le fait que le poème n' aboutisse pas à une véritable fondation. Il nous semble pourtant que les circonstances historiques ne suffisent pas à expliquer cet aspect de l'œuvre, sa réticence envers le mythe : il y a là quelque chose de plus essentiel, une déposition de la métaphysique du pouvoir. La réserve mallarméenne est peut-être une violence étrangère à la problématique du fondement politique. Voir Jacques Rancière, «L'intrus : politique de Mallarmé », Politique de la littérature, 2007, p. 93-112. 
"08-Monginot04" (Col. : RevueRomantisme) — 2011/4/12 — 16:18 — page 16 — \#16 


\title{
Résumés/Abstracts
}

Benoît MONGINOT

\begin{abstract}
"Allégorie et tautologie :
la politique du poème,

de Baudelaire à Mallarmé »

Mallarmé s'oppose à Baudelaire sur une question de poétique. L'allégorie débouche dans ses poèmes sur une mise en relief de la dimension tautologique de tout acte de signification. Nous montrons ici comment cette opposition poétique est un affrontement politique : Mallarmé en redéfinissant l'allégorie redéfinit consciemment les modalités du partage du sens. La pensée de l'allégorie est explicitement chez lui une réflexion sur la communauté, une économie de la violence.
\end{abstract}

Mallarmé is opposed to Baudelaire on a matter of Poetics. In his poems, the use of Allegory entails a magnification of the tautological dimension of every act of signification. This paper sets out to show how this poetical antagonism is politically agonistic: by redefining Allegory, Mallarmé knowingly redefines the workings of meaning-sharing. His thought on Allegory is a conspicuous thinking of the community, an obvious economics of violence. 